Network Working Group

Request for Comments: 4243

Category: Standards Track
M. Stapp

R. Johnson

T. Palaniappan

Cisco Systems, Inc.

December 2005

\title{
Vendor-Specific Information Suboption for the \\ Dynamic Host Configuration Protocol (DHCP) Relay Agent Option
}

\section{Status of This Memo}

This document specifies an Internet standards track protocol for the Internet community, and requests discussion and suggestions for improvements. Please refer to the current edition of the "Internet Official Protocol Standards" (STD 1) for the standardization state and status of this protocol. Distribution of this memo is unlimited.

Copyright Notice

Copyright (C) The Internet Society (2005).

Abstract

This memo defines a new Vendor-Specific Information suboption for the Dynamic Host Configuration Protocol's (DHCP) relay agent information option. The suboption allows a DHCP relay agent to include vendorspecific information in the DHCP messages it forwards, as configured by its administrator.

Table of Contents

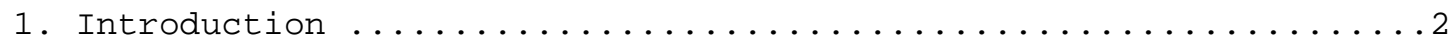

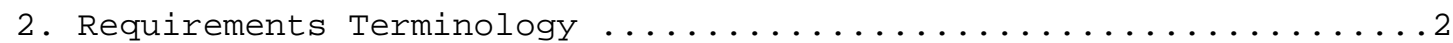

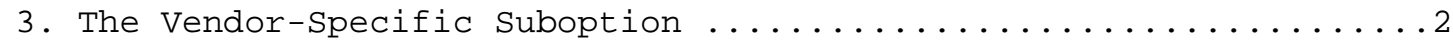

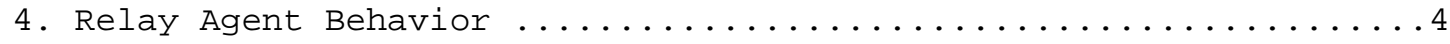

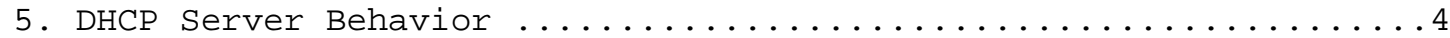

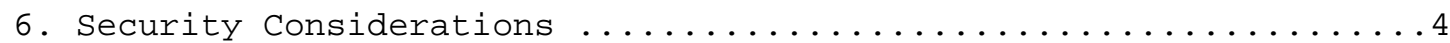

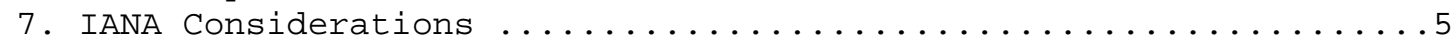

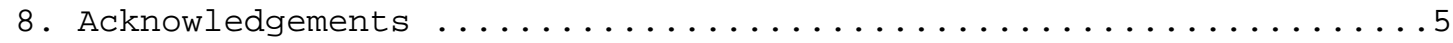

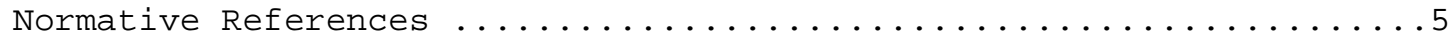

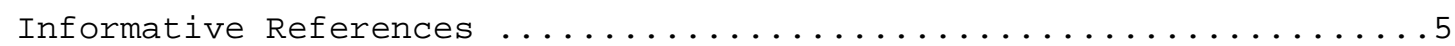




\section{Introduction}

DHCP (RFC 2131 [2]) provides IP addresses and configuration information for IPv4 clients. It includes a relay agent capability, in which processes within the network infrastructure receive broadcast messages from clients and forward them to DHCP servers as unicast messages. In network environments like Docsis data-overcable and xDSL, for example, it has proven useful for the relay agent to add information to the DHCP message before forwarding it, using the relay agent information option (RFC 3046 [3]).

Servers that recognize the relay agent option echo it back in their replies, and some of the information that relays add may be used to help an edge device efficiently return replies to clients. The information that relays supply can also be used in the server's decision making about the addresses and configuration parameters that the client should receive.

In many environments, it's desirable to associate some vendor- or provider-specific information with the clients' DHCP messages. This is often done using the relay agent information option. RFC 3046 defines Remote-ID and Circuit-ID sub-options that are used to carry such information. The values of those suboptions, however, are usually based on some network resource, such as an IP address of a network access device, an ATM Virtual Circuit identifier, or a DocsIS cable-modem identifier. As a result, the values carried in these suboptions are dependent on the physical network configuration. The Vendor-Specific suboption allows administrators to associate other useful data with relayed DHCP messages.

2. Requirements Terminology

The key words "MUST", "MUST NOT", "REQUIRED", "SHALL", "SHALL NOT", "SHOULD", "SHOULD NOT", "RECOMMENDED", "MAY", and "OPTIONAL" in this document are to be interpreted as described in RFC 2119 [1] .

3. The Vendor-Specific Suboption

This memo defines a new DHCP relay agent option suboption that carries vendor-defined data. The suboption takes a form similar to the Vendor-Identifying, Vendor-Specific Option [7]. 


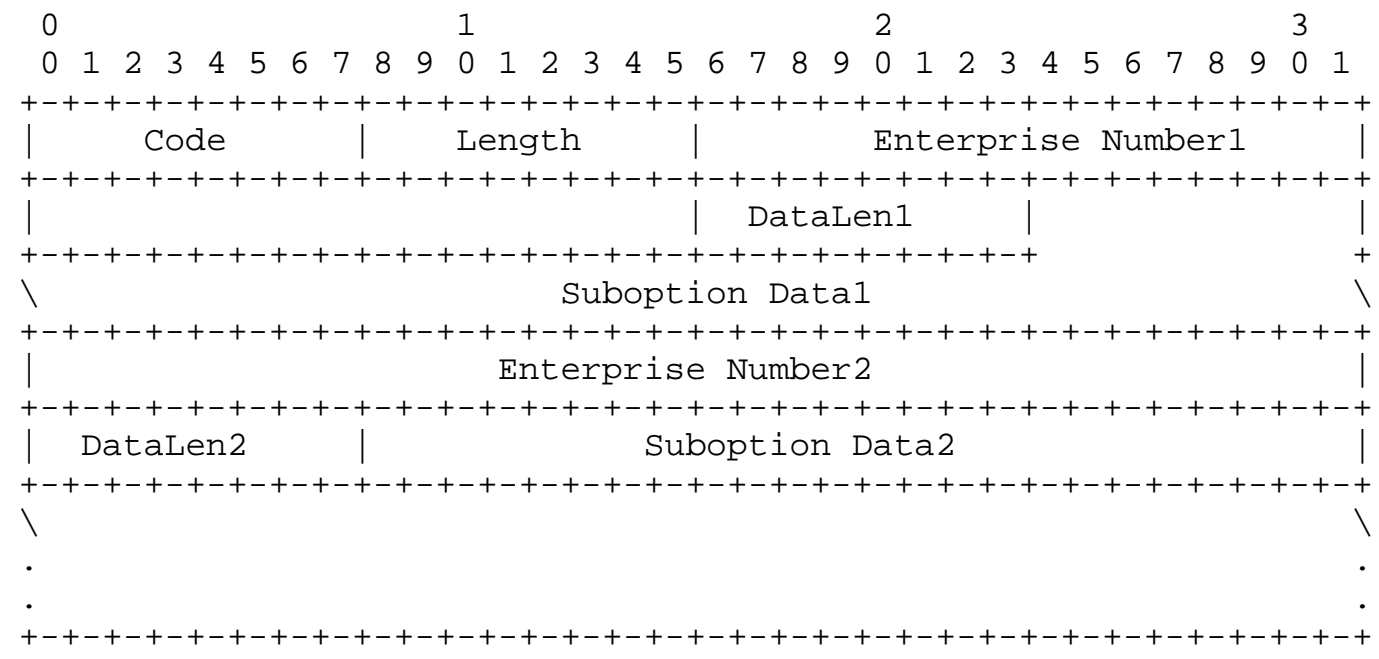

The Code for the suboption is 9.

The one-byte Length field is the length of the data carried in the suboption, in bytes. The length includes the length of the first Enterprise Number; the minimum length is 4 bytes.

"Enterprise NumberN" is a vendor's Enterprise Number as registered with IANA [4]. It is a four-byte integer value in network byteorder.

DataLenN is the length of the data associated with the Enterprise Number.

The Suboption Data is an opaque sequence of bytes.

The Vendor-Specific suboption includes at least one Enterprise Number and carries opaque data defined by the organization identified by the Enterprise Number. A relay may include data associated with more than one vendor's Enterprise Number within a single instance of the Suboption.

Of course, the Vendor-Specific data are vendor-specific. This specification does not establish any requirements on the data in the suboption. Vendors who make use of this suboption are encouraged to document their usage in order to make interoperability possible. 


\section{Relay Agent Behavior}

DHCP relay agents MAY be configured to include Vendor-Specific suboptions if they include a relay agent information option in relayed DHCP messages. The suboptions' types and data are assigned and configured through mechanisms that are outside the scope of this memo.

Relay implementors are encouraged to offer their administrators a means of configuring what data can be included in this suboption, and to document what they are capable of.

5. DHCP Server Behavior

This suboption provides additional information to the DHCP server. The DHCP server, if it is configured to support this suboption, may use this information, in addition to other relay agent option data and other options included in the DHCP client messages, in order to assign an IP address and/or other configuration parameters to the client. There is no special additional processing for this suboption.

6. Security Considerations

Message authentication in DHCP for intradomain use, where the outof-band exchange of a shared secret is feasible, is defined in RFC 3118 [5]. Potential exposures to attack are discussed in section 7 of the DHCP protocol specification in RFC 2131 [2].

The DHCP relay agent option depends on a trusted relationship between the DHCP relay agent and the server, as described in section 5 of RFC 3046. Fraudulent relay agent option data could potentially lead to theft-of-service or exhaustion of limited resources (like IP addresses) by unauthorized clients. A host that tampered with relay agent data associated with another host's DHCP messages could deny service to that host, or interfere with its operation by leading the DHCP server to assign it inappropriate configuration parameters.

While the introduction of fraudulent relay agent options can be prevented by a perimeter defense that blocks these options unless the relay agent is trusted, a deeper defense using authentication for relay agent options via the Authentication Suboption [6] SHOULD be deployed as well.

There are several data in a DHCP message that convey information that may identify an individual host on the network. These include the chaddr, the client-id option, and the hostname and client-fqdn options. Depending on the type of data included, the Vendor-Specific suboption may also convey information that identifies a specific host or a specific user on the network. In practice, this information isn't exposed outside the internal service-provider network, where DHCP messages are usually confined. Administrators who configure data that will be used in DHCP Vendor-Specific suboptions should be careful to use data that are appropriate for the types of networks 
they administer. If DHCP messages travel outside the serviceprovider's own network, or if the suboption values may become visible to other users, it may raise privacy concerns for the access provider or service provider.

7. IANA Considerations

The IANA has assigned the suboption number 9 for the Vendor-Specific Information Suboption from the DHCP Relay Agent Information Option [3] suboption number space.

8. Acknowledgements

The authors are grateful to Andy Sudduth, Josh Littlefield, and Kim Kinnear for their review and comments.

Normative References

[1] Bradner, S., "Key words for use in RFCs to Indicate Requirement Levels", BCP 14, RFC 2119, March 1997.

[2] Droms, R., "Dynamic Host Configuration Protocol", RFC 2131, March 1997.

[3] Patrick, M., "DHCP Relay Agent Information Option", RFC 3046 , January 2001 .

[4] IANA, "Private Enterprise Numbers (http://www.iana.org/ assignments/enterprise-numbers.html)" .

Informative References

[5] Droms, R. and W. Arbaugh, "Authentication for DHCP Messages", RFC 3118, June 2001.

[6] Stapp, M. and T. Lemon, "The Authentication Suboption for the Dynamic Host Configuration Protocol (DHCP) Relay Agent Option", RFC 4030, March 2005.

[7] Littlefield, J., "Vendor-Identifying Vendor Options for Dynamic Host Configuration Protocol version 4 (DHCPv4)", RFC 3925, October 2004 . 


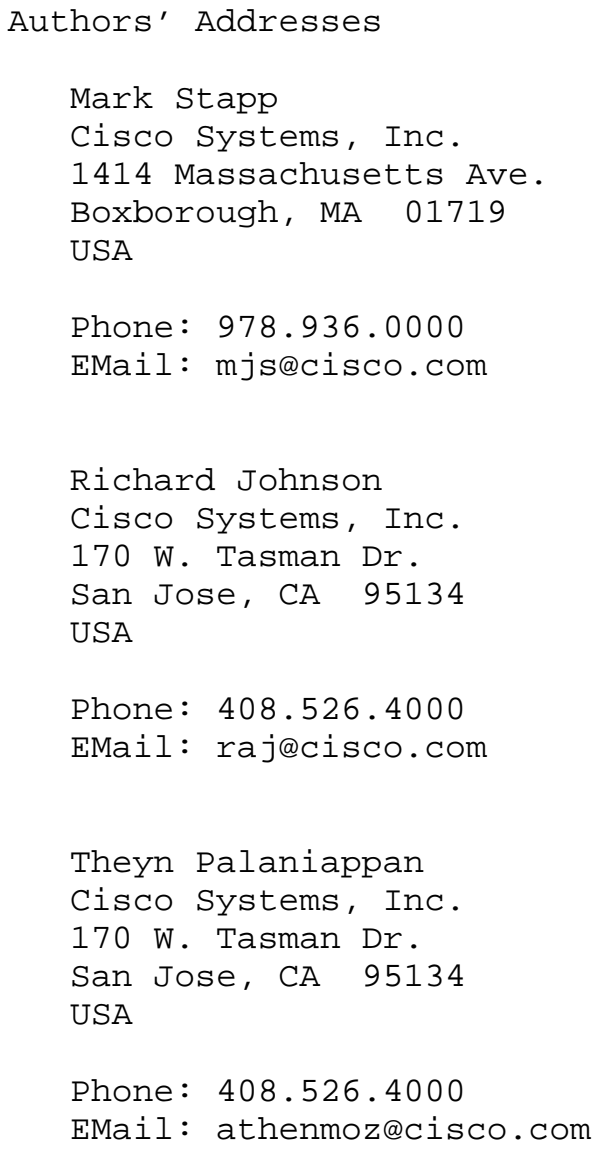


Full Copyright statement

Copyright (C) The Internet Society (2005).

This document is subject to the rights, licenses and restrictions contained in BCP 78, and except as set forth therein, the authors retain all their rights.

This document and the information contained herein are provided on an "AS IS" basis and THE CONTRIBUTOR, THE ORGANIZATION HE/SHE REPRESENTS OR IS SPONSORED BY (IF ANY), THE INTERNET SOCIETY AND THE INTERNET ENGINEERING TASK FORCE DISCLAIM ALL WARRANTIES, EXPRESS OR IMPLIED, INCLUDING BUT NOT LIMITED TO ANY WARRANTY THAT THE USE OF THE INFORMATION HEREIN WILL NOT INFRINGE ANY RIGHTS OR ANY IMPLIED WARRANTIES OF MERCHANTABILITY OR FITNESS FOR A PARTICULAR PURPOSE.

Intellectual Property

The IETF takes no position regarding the validity or scope of any Intellectual Property Rights or other rights that might be claimed to pertain to the implementation or use of the technology described in this document or the extent to which any license under such rights might or might not be available; nor does it represent that it has made any independent effort to identify any such rights. Information on the procedures with respect to rights in RFC documents can be found in BCP 78 and BCP 79 .

Copies of IPR disclosures made to the IETF Secretariat and any assurances of licenses to be made available, or the result of an attempt made to obtain a general license or permission for the use of such proprietary rights by implementers or users of this specification can be obtained from the IETF on-line IPR repository at http://www.ietf.org/ipr.

The IETF invites any interested party to bring to its attention any copyrights, patents or patent applications, or other proprietary rights that may cover technology that may be required to implement this standard. Please address the information to the IETF at ietfipreietf.org.

Acknowledgement

Funding for the RFC Editor function is currently provided by the Internet Society. 